\title{
Certificates of optimality for minimum norm biproportional apportionments
}

\author{
Paolo Serafini
}

\begin{abstract}
Computing a biproportional apportionment that satisfies some given properties may require a high degree of mathematical expertise, that very few voters can share. It seems therefore that the voters have to accept the electoral outcome without any possibility of checking the validity of the stated properties. However, it is possible in some cases to attach to the computed apportionment a certificate which can guarantee the voters of the validity of the apportionment. This type of investigation has been first proposed in [18]. In this paper we pursue the same line of approach and show that a certificate can be produced and easily checked by a layman for apportionments that minimize either an $L_{1}$ - or an $L_{2}$-norm deviation from given quotas.
\end{abstract}

\section{Introduction}

In a recent paper [18] the question was posed of how to 'guarantee' the voters that the seats assigned by the competent authority to parties and constituencies are consistent with certain properties the apportionment is claimed to satisfy.

The question was originated by the fact that usually electoral systems have to rely on complex mathematical procedures to find an apportionment. Indeed, naive and 'easy to understand' procedures may generate inconsistencies and anomalies [3,10-13]. On the other hand transparency of the electoral machinery is a fundamental property because not only the citizens should be able to easily check the correctness of the seat assignments (Principle 1 in the so-called "Erice Decalogue" [19]) but also the electoral authority in first place.

In [18] the following dilemma was stated: 'Which is better? To have simple, but unsound electoral laws, or sound, but complex ones?' The proposed way out of the dilemma was: 'Leave to a mathematically sophisticated algorithm the task of producing a sound solution, but attach to it a certificate of guarantee, that is, describe a simple procedure whereby anybody can check, through some elementary operations, that the solution output by the algorithm indeed satisfies all the requirements sought for.'

What we mean is that this 'anybody' does not need to be a mathematician who only can understand how the mathematical principles are embodied in an algorithm, nor a computer scientists who only can verify the correctness of the algorithm implementation. Only an elementary knowledge of mathematics and the ability of understanding simple mathematical arguments should be required for the verification procedure.

The specific electoral system addressed by [18] was the biproportional apportionment problem that minimizes an $L_{\infty}$-norm deviation from given quotas (this type of apportionment has been fully investigated in [17]). In that paper it was shown that it is possible to attach to an apportionment a certificate of guarantee that anybody can check with only an elementary knowledge of mathematics.

Choosing a different norm leads usually to a different apportionment (see for instance [16] for a review). It is matter of the law-maker to choose a particular measure of deviation. In case a norm different from the $L_{\infty}$-norm is adopted, it would be interesting to see whether also different norms are amenable to a certificate and a checking procedure.

In this paper we continue the type of investigation started in [18] and show that a similar certificate can be indeed attached also to apportionments satisfying different measures of deviation from given quotas. In particular we consider the Controlled rounding procedure [4], i.e., an $L_{2}$-norm minimization within quotas, and the $L_{1}$-norm minimization (without restrictions).

This is possible because these minimization problems, although requiring integrality of the apportionment, do satisfy the strong duality property of mathematical programming. Hence verification is simply a strong 
duality check. The problem we are concerned with in this paper is to make this check easy to be understood and carried out without any knowledge of duality properties nor of the mathematics of optimization problems.

Of course we assume that readers of this paper are acquainted with linear programming and duality properties. Therefore we take these concepts for granted. Otherwise a comprehensive reference, among many others, can be found in [7].

The paper is organized as follows. In Section 2 we formally define the problem we are dealing with. In Section 3 we provide some explanations of what we intend for certificate. Then in Section 4 we build a certification scheme for the Controlled rounding procedure [4], i.e., an $L_{2}$-norm minimization without quota violation, and in Section 5 we do the same for the $L_{1}$-norm minimization. One example is provided in Section 6 to illustrate in detail the proposed certification. Conclusions follow in Section 7.

\section{Problem statement}

The biproportional apportionment problem (BAP) is the following problem. There is a set $M$ of electoral constituencies and a set $N$ of parties. Before the elections each constituency $i \in M$ is assigned $r_{i}$ seats, with $H=\sum_{i \in M} r_{i}$ being the house size. After the elections each party $j \in N$ is assigned $p_{j}$ seats in the house according to the votes received at national level. Let $v_{i j}$ be the votes received by party $j$ in constituency $i$. From these data one has to compute the number $x_{i j}$ of seats to be assigned to party $j$ in constituency $i$ such that:

$$
\sum_{j \in N} x_{i j}=r_{i}, i \in M, \quad \sum_{i \in M} x_{i j}=p_{j}, j \in N, \quad x_{i j}=0 \text { if } v_{i j}=0
$$

and the seats $x_{i j}$ are as proportional as possible to the votes $v_{i j}$. Clearly this requirement needs a formal specification. The way this is done leads to different approaches in the literature.

Basically there are two approaches to BAP. One approach is based on characterizing proportionality via a set of axioms and finding the unique apportionment that satisfies them $[1,2,14,8,5,6]$. Another approach is based on finding an apportionment that minimizes a measure of deviation from given quotas $[4,15-17]$.

In this paper we consider the latter approach. We recall that quotas represent an ideal apportionment with just the integrality requirement relaxed. Perhaps the most important quotas are the so called fair share quotas introduced by [1] which meet the proportionality axioms for fractional apportionments. The fair share quotas are defined via multipliers $\lambda_{i}>0$ and $\mu_{j}>0$ such that

$$
q_{i j}=\lambda_{i} v_{i j} \mu_{j}
$$

and

$$
\sum_{j \in N} q_{i j}=r_{i}, \quad i \in M, \quad \sum_{i \in M} q_{i j}=p_{j}, \quad j \in N
$$

A full characterization of fair share quotas can be found in $[1,2,9]$. Sometimes different quotas are used in real electoral systems (e.g. Italy, Belgium) like the so called regional quotas:

$$
q_{i j}=\frac{v_{i j}}{\sum_{k \in J} v_{i k}} r_{i}, \quad j \in N, i \in M
$$

Regional quotas have the disadvantage that in general $\sum_{i \in M} q_{i j} \neq p_{j}$ (but clearly $\sum_{j \in N} q_{i j}=r_{i}$ ). On the other hand they offer the advantage that quotas in one constituency are independent of the votes in another constituency and this can be perceived as a desirable feature when autonomy of different constituencies has to be preserved. 
No matter how quotas are defined the minimization approach consists in finding the apportionment that minimizes the quantity $\|x-q\|$. In [18] the possibility of a certificate for an $L_{\infty}$-norm was investigated. In this paper we pursue the same goal for the $L_{2}$ - and the $L_{1}$-norm. More exactly, we consider the $L_{2}$-norm with the added requirement that the apportionment respects the quotas, i.e. $x_{i j} \in\left\{\left\lfloor q_{i j}\right\rfloor,\left\lceil q_{i j}\right\rceil\right\}$, the so-called Controlled rounding method [4] while for the $L_{1}$-norm we do not have any restriction.

\section{The meaning of 'certificate'}

Let us call 'Solver' the person who actually solves BAP and claims that the computed apportionment is the one that makes minimum the following quantity

$$
\sum_{i j}\left|x_{i j}-q_{i j}\right|^{p}
$$

where $p=1$ or $p=2$ according to the choice.

Let us call 'Verifier' the person who has to check the validity of this claim. To this aim the Solver has to provide the Verifier also with a certificate, that, together with the apportionment, allows the Verifier to carry out the check.

The idea of a 'certificate' is clearly borrowed from the definition of the class NP in computational complexity. However, beside proving the claim, we also require that the verification procedure be simple enough to be carried out by a person with an elementary knowledge of mathematics.

Indeed we only require the Verifier to be able to carry out ordinary sums and subtractions and to know the meaning of $|a|,\lfloor a\rfloor,\lceil a\rceil$ and $\langle a\rangle$ (the fractional part) for a given number $a$ (notions that can be easily learned on the spot). The Verifier has to make these computations on the solution and on the certificate.

The certificate consists of $|M|+|N|$ numbers $u_{i}, i \in M$, and $w_{j}, j \in N$. Equipped with these numbers and following a simple argument which will presented in the next section the Verifier makes a computation and at the end of it he/she has to conclude that the apportionment is indeed the one that minimizes (1).

It will be clear to the readers that the certificate is nothing but the dual variables of the problem and that the verification procedure is just a check of the complementarity condition. However, we have to keep in mind that the Verifier is not supposed to know anything about linear programming and duality properties. Hence we have to provide the Verifier with simple yet convincing arguments which mimic the complementarity conditions. This is the aim of the next two sections.

\section{A certificate for the Controlled rounding apportionment}

In the Controlled rounding procedure $[4,16]$ the apportionment $x_{i j}$ must satisfy $x_{i j} \in\left\{\left\lfloor q_{i j}\right\rfloor,\left\lceil q_{i j}\right\rceil\right\}$. Hence the problem may be rephrased by saying that one has to compute a binary matrix $y_{i j} \in\{0,1\}$ that must satisfy the constraints

$$
\sum_{i \in M} y_{i j}=p_{j}-\sum_{i \in M}\left\lfloor q_{i j}\right\rfloor=: \tilde{p}_{j}, j \in N, \quad \sum_{j \in N} y_{i j}=r_{i}-\sum_{j \in N}\left\lfloor q_{i j}\right\rfloor=: \tilde{r}_{i} i \in M
$$

and the apportionment is given by $x_{i j}=\left\lfloor q_{i j}\right\rfloor+y_{i j}$. The Verifier is therefore given a 'table' $y$ of zeros and ones, with the property that in row $i$ there must be $\tilde{r}_{i}$ ones and in column $j$ there must be $\tilde{p}_{j}$ ones. A table satisfying this property is said to be feasible. 
The proof that the solution provided by the Solver is indeed the optimal one consists of the following arguments which we may divide into four steps. Of course the Verifier has to be convinced of the logical validity of each step.

The very first step the Verifier has to understand is that an apportionment can be framed as a feasible table $y$ of zeros and ones, once we have already assigned $\left\lfloor q_{i j}\right\rfloor$ seats. Plainly speaking, for the pairs $(i, j)$ where there is a one, $q_{i j}$ has to be rounded up and for the pairs $(i, j)$ where there is a zero, $q_{i j}$ has to be rounded down.

In the second step the Verifier has to understand that there is a 'cost' in assigning a one to the $(i, j)$ entry in the table instead of leaving the zero. According to (1), leaving the zero corresponds to a deviation from the quotas given by

$$
\left(q_{i j}-\left\lfloor q_{i j}\right\rfloor\right)^{p}=\left(\left\langle q_{i j}\right\rangle\right)^{p}
$$

whereas putting a one corresponds to a deviation from the quotas given by

$$
\left(\left\lceil q_{i j}\right\rceil-q_{i j}\right)^{p}=\left(1-\left\langle q_{i j}\right\rangle\right)^{p}
$$

Hence the difference between putting a one and leaving a zero, i.e., the 'cost' $c_{i j}$ of putting a one, is

$$
c_{i j}:=\left(1-\left\langle q_{i j}\right\rangle\right)^{p}-\left(\left\langle q_{i j}\right\rangle\right)^{p}
$$

which, for both $p=1$ and $p=2$, is

$$
c_{i j}=1-2\left\langle q_{i j}\right\rangle
$$

It is evident that the cost can be negative, in which case putting a one gives a premium, whereas it gives a penalty when it is positive.

At this point it should be be clear to the Verifier that the apportionment $x$ yields the minimum value of (1) if and only if the table $y$ is the one which yields the minimum value of $\sum_{i j} c_{i j} y_{i j}$. Hence the Verifier has to check this second claim.

To this purpose the third step for the Verifier is to understand that the problem is just the same if the costs in one row are all increased (or decreased) by the same quantity. Since the number of ones to be put in a row is invariant for all feasible tables, the costs of all feasible tables change by the same quantity and therefore the optimal table for the original costs must be optimal also for the modified costs. The same observation is valid for the columns.

Therefore, if the Solver provides numbers $u_{i}$ and $w_{j}$, the optimal table for the costs (which we clearly recognize as the reduced costs)

$$
c_{i j}^{\prime}=c_{i j}-u_{i}-w_{j}
$$

is also optimal for the original costs $c_{i j}$.

The fourth step the Verifier has to understand is the following argument: if, for a given table $y$ (not necessarily feasible), in each place where there is a one the cost is null or negative and in each place where there is a zero the cost is null or positive, then there can be no better table. Indeed the minimum value of the expression

$$
\sum_{i, j} c_{i j} y_{i j}
$$

for any value of $y_{i j} \in\{0,1\}$, disregarding the constraints $\sum_{i} y_{i j}=\tilde{p}_{j}$ and $\sum_{j} y_{i j}=\tilde{r}_{i}$, is attained for $y_{i j}=1$ if $c_{i j}<0$ and $y_{i j}=0$ if $c_{i j}>0$. If $c_{i j}=0, y_{i j}$ can be either one or zero. If, in addition, these values do satisfy the sum constraints, then they are necessarily the best table and the apportionment $x_{i j}=\left\lfloor q_{i j}\right\rfloor+y_{i j}$ is optimal. 
Of course we do not expect that the costs (2) and the table $y$ given by the Solver satisfy this property. However, if the apportionment is the optimal one it is always possible to modify the costs into the reduced costs $c^{\prime}$, in such a way that the property is satisfied. The existence of values $u_{i}$ and $w_{j}$ such that $y$ is optimal if and only if

$$
c_{i j}-u_{i}-w_{j}<0 \Longrightarrow y_{i j}=1, \quad c_{i j}-u_{i}-w_{j}>0 \Longrightarrow y_{i j}=0
$$

is guaranteed by the complementarity theorem of linear programming. We stress the fact that the Verifier has to know nothing about this theorem and strong duality properties, let alone linear programming.

Summing up, the Solver gives the Verifier the table $y$ and the numbers $u_{i}$ e $w_{j}$, that constitute the certificate. The Verifier has only to be convinced that a table $y$ optimal for the costs $c_{i j}$ yields an optimal apportionment with respect to (1), and that a table optimal for the $\operatorname{costs} c_{i j}$ is also optimal for the $\operatorname{costs} c_{i j}^{\prime}$ (independently of the values $u_{i}$ and $w_{j}$ ) and finally that the conditions (3) guarantee optimality.

We admit that the four steps we have listed are not amenable to a person without any mathematical background. However, they do not require any specific knowledge of some particular branch of mathematics, just a minimum acquaintance with logical reasoning, and therefore can be carried out by an acculturated layman.

\section{A certificate for $L_{1}$-norm minimal apportionments}

A similar certificate, slightly more complicated, can be given also for apportionments that minimize the $L_{1}$-norm, without the constraint of non violating the quotas.

In this case we need three tables $y^{1}, y^{2}$ and $y^{3}$. The tables are constrained as follows

$$
y_{i j}^{1} \in\left\{0,1, \ldots,\left\lfloor q_{i j}\right\rfloor\right\}, \quad y_{i j}^{2} \in\{0,1\}, \quad y_{i j}^{3} \in\{0,1,2, \ldots\} .
$$

Furthermore,

$$
\begin{array}{ll}
\text { if } \quad y_{i j}^{1}<\left\lfloor q_{i j}\right\rfloor & \text { then both } y_{i j}^{2}=0 \text { and } y_{i j}^{1}=0, \\
\text { if } \quad y_{i j}^{2}=0 \quad \text { then } y_{i j}^{1}=0 . &
\end{array}
$$

In other words $y_{i j}^{2}$ can be positive only if $y_{i j}^{1}$ is equal to its maximum value $\left\lfloor q_{i j}\right\rfloor$ and $y_{i j}^{3}$ can be positive only if $y_{i j}^{2}=1$ (and a fortiori also $y_{i j}^{1}=\left\lfloor q_{i j}\right\rfloor$ ). The apportionment is given by

$$
x_{i j}=y_{i j}^{1}+y_{i j}^{2}+y_{i j}^{3}
$$

Note that each $y_{i j}^{3}$ in (4) is implicitly upper bounded by $\min \left\{r_{i}, p_{j}\right\}-\left\lceil q_{i j}\right\rceil$. The rationale behind the constraints (4) and (5) is that the number of seats $x_{i j}$ is formed by first assigning a positive value to $y_{i j}^{1}$ up to the bound $\left\lfloor q_{i j}\right\rfloor$ if necessary. If $x_{i j}$ has to be greater than $\left\lfloor q_{i j}\right\rfloor$ then the additional seat is taken care of by $y_{i j}^{2}$. Finally if $x_{i j}$ has to be greater than $\left\lceil q_{i j}\right\rceil$ the extra seats are taken care of by $y_{i j}^{3}$.

There are costs associated to the three tables induced by the objective function

$$
\sum_{i j}\left|x_{i j}-q_{i j}\right|
$$

The costs are evaluated as follows. If $x_{i j}=0$ the apportionment value is $q_{i j}$, as from (6). Starting from zero and up to the value $\left\lfloor q_{i j}\right\rfloor$ each additional seat assigned to the pair $(i, j)$ decrements the cost by 1 . Indeed, if $x_{i j} \leq\left\lfloor q_{i j}\right\rfloor$ we have

$$
c_{i j}^{1}=\left|x_{i j}-q_{i j}\right|-\left|x_{i j}-1-q_{i j}\right|=\left(q_{i j}-x_{i j}\right)-\left(q_{i j}-x_{i j}+1\right)=-1
$$


Since $x_{i j} \leq\left\lfloor q_{i j}\right\rfloor$ we have $x_{i j}=y_{i j}^{1}$ and therefore the cost $c_{i j}^{1}$ has to be attributed to $y_{i j}^{1}$. Once there are $\left\lfloor q_{i j}\right\rfloor$ seats for the pair $(i, j)$ (whose cost $q_{i j}-\left\lfloor q_{i j}\right\rfloor$ is taken care of by the value $y_{i j}^{1}=\left\lfloor q_{i j}\right\rfloor$ ) an additional seat, which corresponds to setting $y_{i j}^{2}=1$, costs

$$
c_{i j}^{2}=\left|\left\lceil q_{i j}\right\rceil-q_{i j}\right|-\left|\left\lfloor q_{i j}\right\rfloor-q_{i j}\right|=1-2\left\langle q_{i j}\right\rangle
$$

and the $\operatorname{cost} c_{i j}^{2}$ has to be attributed to $y_{i j}^{2}$. Then, once $x_{i j}=\left\lceil q_{i j}\right\rceil$ (whose cost $1-\left\langle q_{i j}\right\rangle$ is taken care of by the value $y_{i j}^{1}=\left\lfloor q_{i j}\right\rfloor$ and $\left.y_{i j}^{2}=1\right)$ each additional seats costs 1 , as can be seen from

$$
c_{i j}^{3}=\left|x_{i j}+1-q_{i j}\right|-\left|x_{i j}-q_{i j}\right|=\left(x_{i j}+1-q_{i j}\right)-\left(x_{i j}-q_{i j}\right)=1
$$

and the cost $c_{i j}^{3}$ has to be attributed to $y_{i j}^{3}$.

Now the invariance on the number of seats to be apportioned is related to the three tables together. Hence the problem is not changed if the $\operatorname{costs} c_{i j}^{1}, c_{i j}^{2}$ and $c_{i j}^{3}$ are simultaneously increased or decreased by the same quantity in a particular row $i$. The same is true if the costs are simultaneously increased or decreased by the same quantity in a particular column $j$. Therefore the Verifier has to understand that, given values $u_{i}$ and $w_{j}$, the costs can be modified into the reduced costs

$$
c_{i j}^{\prime 1}=-1-u_{i}-w_{j}, \quad c_{i j}^{\prime 2}=1-2\left\langle q_{i j}\right\rangle-u_{i}-w_{j}, \quad c_{i j}^{\prime 3}=1-u_{i}-w_{j}
$$

without modifying the problem, i.e., leaving invariant the optimal apportionment.

Finally the Verifier must be convinced that the tables $y^{1}, y^{2}$ and $y^{3}$ are optimal for the costs $c^{\prime 1}, c^{\prime 2}$ and $c^{\prime 3}$ respectively (and therefore also for the costs $c^{1}, c^{2}$ and $c^{3}$ ) if

$$
\begin{aligned}
& c_{i j}^{\prime 1}<0 \quad \Longrightarrow \quad y_{i j}^{1}=\left\lfloor q_{i j}\right\rfloor, \quad c_{i j}^{\prime 1}>0 \quad \Longrightarrow \quad y_{i j}^{1}=0 \\
& c_{i j}^{\prime 2}<0 \quad \Longrightarrow \quad y_{i j}^{2}=1, \quad c_{i j}^{\prime 2}>0 \quad \Longrightarrow \quad y_{i j}^{2}=0 \\
& c_{i j}^{\prime 3}<0 \quad \Longrightarrow \quad y_{i j}^{3}=r_{i}-\left\lceil q_{i j}\right\rceil, \quad c_{i j}^{\prime 3}>0 \quad \Longrightarrow \quad y_{i j}^{3}=0
\end{aligned}
$$

The reason is clear and is based on the same arguments as for the Controlled rounding procedure: the best possible tables (disregarding the sum constraints) are the ones whose entries are at the upper bound in correspondence of a negative cost and at the lower bound in correspondence of a positive cost. If the tables obey the sum constraints and the constraints (4) and (5) they necessarily constitute the best apportionment.

\section{Examples}

Let us assume that the electoral data are

$$
\begin{aligned}
v & =\left(\begin{array}{rrrr}
10356 & 6997 & 8380 & 11040 \\
16547 & 4165 & 9987 & 10710 \\
1697 & 4493 & 13880 & 12043 \\
24465 & 27132 & 28203 & 19594 \\
42350 & 575 & 6451 & 4476
\end{array}\right), \quad r=\left(\begin{array}{l}
16 \\
20 \\
16 \\
21 \\
27
\end{array}\right) \\
p & =\left(\begin{array}{rrrr}
36 & 17 & 25 & 22
\end{array}\right)
\end{aligned}
$$


For these data the fair share quotas are (all figures in this section are displayed rounded to three decimal digits)

$$
q=\left(\begin{array}{rrrr}
3.769 & 3.914 & 3.655 & 4.662 \\
6.990 & 2.705 & 5.056 & 5.249 \\
0.692 & 2.818 & 6.788 & 5.702 \\
4.187 & 7.138 & 5.784 & 3.891 \\
20.361 & 0.425 & 3.717 & 2.497
\end{array}\right)
$$

Suppose we want to find the best apportionment with respect to the fair share quotas according to the Controlled rounding procedure. So the Solver produces the following apportionment

$$
x=\lfloor q\rfloor+y=\left(\begin{array}{rrrr}
3 & 3 & 3 & 4 \\
6 & 2 & 5 & 5 \\
0 & 2 & 6 & 5 \\
4 & 7 & 5 & 3 \\
20 & 0 & 3 & 2
\end{array}\right)+\left(\begin{array}{llll}
1 & 1 & 0 & 1 \\
1 & 1 & 0 & 0 \\
1 & 1 & 1 & 0 \\
0 & 0 & 1 & 1 \\
0 & 0 & 1 & 1
\end{array}\right)=\left(\begin{array}{rrrr}
4 & 4 & 3 & 5 \\
7 & 3 & 5 & 5 \\
1 & 3 & 7 & 5 \\
4 & 7 & 6 & 4 \\
20 & 0 & 4 & 3
\end{array}\right)
$$

together with the certificate

$$
u=\left(\begin{array}{lllll}
-0.144 & 0 & -0.410 & 0 & 0
\end{array}\right), \quad w=\left(\begin{array}{llll}
0.025 & -0.227 & -0.166 & 0.007
\end{array}\right)
$$

To check that it is indeed the best apportionment the Verifier first computes the costs, according to (2)

$$
c=\left(\begin{array}{rrrr}
-0.538 & -0.829 & -0.310 & -0.323 \\
-0.980 & -0.409 & 0.888 & 0.502 \\
-0.385 & -0.637 & -0.575 & -0.403 \\
0.626 & 0.724 & -0.569 & -0.781 \\
0.278 & 0.150 & -0.434 & 0.007
\end{array}\right)
$$

Then by using the certificate the Verifier computes the reduced costs

$$
c^{\prime}=\left(\begin{array}{rrrr}
-0.419 & -0.458 & 0 & -0.185 \\
-1.005 & -0.182 & 1.054 & 0.495 \\
0 & 0 & 0 & 0 \\
0.601 & 0.951 & -0.403 & -0.788 \\
0.253 & 0.377 & -0.268 & 0
\end{array}\right)
$$

It remains to check that wherever $c_{i j}^{\prime}>0$ we have $y_{i j}=0$ and wherever $c_{i j}^{\prime}<0$ we have $y_{i j}=1$. Since this is true the Verifier has the proof that the apportionment is optimal.

Let us now suppose that we want to find the best apportionment with respect to the regional quotas for the $L_{1}$-norm without the constraint of non violating the quotas. The regional quotas for the same electoral data are

$$
q=\left(\begin{array}{rrrr}
4.506 & 3.044 & 3.646 & 4.804 \\
7.992 & 2.012 & 4.824 & 5.173 \\
0.846 & 2.239 & 6.916 & 6.000 \\
5.169 & 5.732 & 5.959 & 4.140 \\
21.233 & 0.288 & 3.234 & 2.244
\end{array}\right)
$$


So the Solver produces the following apportionment

$$
\begin{gathered}
x=y^{1}+y^{2}+y^{3}=\left(\begin{array}{rrrr}
3 & 3 & 3 & 4 \\
7 & 2 & 4 & 5 \\
0 & 2 & 6 & 6 \\
5 & 5 & 5 & 4 \\
21 & 0 & 3 & 2
\end{array}\right)+\left(\begin{array}{llll}
0 & 1 & 1 & 1 \\
0 & 1 & 1 & 0 \\
0 & 1 & 1 & 0 \\
0 & 1 & 1 & 0 \\
0 & 1 & 0 & 0
\end{array}\right)+\left(\begin{array}{llll}
0 & 0 & 0 & 0 \\
0 & 0 & 0 & 0 \\
0 & 0 & 0 & 0 \\
0 & 0 & 0 & 0 \\
0 & 0 & 0 & 0
\end{array}\right) \\
x=\left(\begin{array}{rrrr}
3 & 4 & 4 & 5 \\
7 & 3 & 5 & 5 \\
0 & 3 & 7 & 6 \\
5 & 6 & 6 & 4 \\
21 & 1 & 3 & 2
\end{array}\right)
\end{gathered}
$$

together with the certificate

$$
u=\left(\begin{array}{lllll}
0.477 & 0.477 & 0 . & 0.477 & 0.477
\end{array}\right), \quad w=\left(\begin{array}{llll}
-1.477 & 0.523 & -0.769 & -1 .
\end{array}\right)
$$

The Verifier first checks that the tables $y^{1}, y^{2}$ and $y^{3}$ are consistent with the constraints (4) and (5). Then the Verifier computes the costs $c^{2}$ according to (7) ( $c^{1}$ and $c^{3}$ are predetermined), as

$$
c^{2}=1-2\langle q\rangle=\left(\begin{array}{rrrr}
-0.012 & 0.911 & -0.292 & -0.607 \\
-0.984 & 0.977 & -0.647 & 0.654 \\
-0.691 & 0.523 & -0.831 & 0.999 \\
0.662 & -0.465 & -0.917 & 0.720 \\
0.534 & 0.423 & 0.531 & 0.512
\end{array}\right) \text {, }
$$

Then the Verifier computes the reduced costs

$$
\begin{aligned}
c^{\prime 1}= & \left(\begin{array}{rrrr}
0 & -2.000 & -0.708 & -0.477 \\
0 & -2.000 & -0.708 & -0.477 \\
0.477 & -1.523 & -0.231 & 0 \\
0 & -2.000 & -0.708 & -0.477 \\
0 & -2.000 & -0.708 & -0.477
\end{array}\right) \\
c^{\prime 2} & =\left(\begin{array}{rrrr}
0.988 & -0.089 & 0 & -0.084 \\
0.016 & -0.023 & -0.355 & 1.177 \\
0.786 & 0 & -0.062 & 1.999 \\
1.662 & -1.465 & -0.625 & 1.243 \\
1.534 & -0.577 & 0.824 & 1.035
\end{array}\right) \\
c^{\prime 3} & =\left(\begin{array}{rrrr}
2.000 & 0 & 1.292 & 1.523 \\
2.000 & 0 & 1.292 & 1.523 \\
2.477 & 0.477 & 1.769 & 2.000 \\
2.000 & 0 & 1.292 & 1.523 \\
2.000 & 0 & 1.292 & 1.523
\end{array}\right)
\end{aligned}
$$

Finally the Verifier can check that the conditions (8) are met and therefore the apportionment is optimal. 


\section{Conclusions}

In this paper we have tried to solve the conflict between accuracy and transparency for the computation of biproportional apportionments in case the apportionment has to satisfy a minimum deviation from assigned quotas. A mathematical procedure to find the best apportionment has to be necessarily complex and only expert mathematicians can design the correct procedure and produce the required apportionment. These procedures, not only are too complex to be written down in an electoral law, but they can raise doubts on the validity of the final outcome not only for the vast majority of people that are not familiar with mathematical techniques but also for the authority that has the legal power to confirm the validity of the apportionment.

It is therefore mandatory to provide the possibility of checking the solution without repeating the computation but only with a simple computation requiring elementary mathematics and a basic reasoning. Trying to find this kind of checking was the subject of the paper [18] which dealt with apportionments minimizing an $L_{\infty}$-norm deviation. In this paper we have continued the investigation extending the possibility of checking also to $L_{1^{-}}$and $L_{2}$-norm deviation.

Since the related mathematical programming problems turn to have the strong duality property, the check is indeed possible and is essentially based on the complementarity relations. Obviously the layman is not required to have this deep mathematical knowledge. Our challenge was to rephrase strong duality and complementarity conditions in a down-to-earth way with a minimum of mathematical background.

As observed in [18] "the adoption of a certificate of guarantee in presence of complex electoral rules which cannot be easily replicated by the layman, may result in better quality electoral systems without prejudice of transparency."

\section{References}

1. M. Balinski and G. Demange: An axiomatic approach to proportionality between matrices, Mathematics of Operations Research, 14, 700-719 (1989).

2. M. Balinski and G. Demange: Algorithms for proportional matrices in reals and integers, Mathematical Programming, 45, 193-210 (1989).

3. M.L. Balinski and V. Ramírez: Mexican Electoral Law: 1996 version, Electoral Studies, 16, 329-349 (1997).

4. L.H. Cox and L.R. Ernst: Controlled rounding, INFOR - Information Systems and Operational Research, 20, 423-432 (1982).

5. N. Gaffke and F. Pukelsheim: Divisor methods for proportional representation systems: An optimization approach to vector and matrix apportionment problems, Mathematical Social Sciences, 56, 166-184 (2008).

6. N. Gaffke and F. Pukelsheim: Vector and matrix apportionment problems and separable convex integer optimization, Mathematical Methods of Operations Research, 67, 133-159 (2008).

7. F.S. Hillier and G.J. Liebermann: Introduction to operations research. Mc Graw Hill (2010).

8. S. Maier and F. Pukelsheim: Bazi: A Free Computer Program for Proportional Representation Apportionment. Preprint Nr. 042/2007. Institut für Mathematik, Universität Augsburg, 2007. Internet: www. opus-bayern.de/uni-augsburg/volltexte/2007/711/ (2007). 
9. B. Kalantari, I. Lari, F. Ricca and B. Simeone: On the complexity of general matrix scaling and entropy minimization via the RAS algorithm, Mathematical Programming, Series A, 112, 371-401 (2008).

10. Pennisi A.: The Italian bug: a flawed procedure for bi-proportional seat allocation, in: B. Simeone, F. Pukelsheim (eds), Mathematics and democracy: Recent advances in voting systems and collective choice, pp. 151-166, Berlin, Springer (2006).

11. Pennisi A., F. Ricca, B. Simeone: Malfunzionamenti dell'allocazione biproporzionale di seggi nella riforma elettorale italiana. Dipartimento di Statistica, Probabilità e Statistiche Applicate, Università La Sapienza, Roma, Serie A - Ricerche, n. 21 (2005).

12. Pennisi A., F. Ricca, B. Simeone: Legge elettorale con paradosso. La Voce, 11 Novembre (2005).

13. Pennisi A., F. Ricca, B. Simeone: Bachi e buchi della legge elettorale italiana nell'allocazione biproporzionale di seggi, Sociologia e Ricerca Sociale, 79, 55-76 (2006).

14. F. Pukelsheim: BAZI: A Java program for proportional representation. Oberwolfach Reports 1, 735-737, www .uni-augsburg.de/bazi (2004).

15. F. Pukelsheim, F. Ricca, A. Scozzari, P. Serafini, and B. Simeone: Network flow methods for electoral systems, Networks, 59, 73-88, DOI: 10.1002/net.20480 (2012).

16. F. Ricca, A. Scozzari, P. Serafini and B. Simeone: Error minimization methods in biproportional apportionment, TOP, 20, 547-577 (2012).

17. P. Serafini and B. Simeone: Parametric maximum flow methods for minimax approximation of target quotas in biproportional apportionment, Networks, 59, 191-208 (2012).

18. P. Serafini and B. Simeone: Certificates of optimality: the third way to biproportional apportionment, Social Choice and Welfare, 38, 247-268 (2012).

19. B. Simeone, F. Pukelsheim (eds.): Mathematics and Democracy. Recent Advances in Voting Systems and Collective Choice. Studies in Choice and Welfare, Springer (2006). 